\title{
Plan d'améliorations communautaires : Comment assurer la réussite?
}

\begin{abstract}
Vanessa Carrière
Dans cette recherche, il sera expliqué comment le gouvernement municipal peut assurer une implantation réussie de son Plan d'améliorations communautaires (PAC) pour ainsi attirer plus d'entreprises, de résidents et de touristes. Compléter la littérature d'un projet est une étape de franchie, par contre si les mesures d'implantation ne sont pas suivies, il sera impossible d'assurer sa réussite. Depuis janvier 2016, la municipalité de Russell offre le programme, par contre des améliorations doivent être apportées pour assurer une mise en œuvre plus efficace. Les étapes essentielles étant la promotion, l'observation, l'exécution et la révision seront détaillées par l'entremise d'études antérieures et d'études de cas réelles. Selon le Plan stratégique de développement économique 2014-2016 de la municipalité de Russell, l'un des objectifs pour soutenir la croissance de la communauté est de « promouvoir l'attrait de la population par le biais d'un développement urbain bien planifié, en phase avec les stratégies de Prescott-Russell pour fonder un lieu d'une qualité exceptionnelle » (Municipalité de Russell, 2015). Une mise en œuvre du Plan d'améliorations communautaires fondée sur les éléments essentiels à la réussite viendra certainement améliorer une communauté pour le meilleur.
\end{abstract}

Mots-clés : Plan d'améliorations communautaires, programme incitatif, municipalité, développement économique

\section{Introduction}

Dans ce projet, il sera expliqué comment le gouvernement municipal peut assurer une implantation réussie de son Plan d'améliorations communautaires (PAC) pour ainsi attirer plus d'entreprises, de résidents et de touristes. Compléter la littérature d'un projet est une étape de franchie, par contre si les mesures d'implantation ne sont pas suivies, il sera impossible d'assurer sa réussite. Depuis janvier 2016, la municipalité de Russell offre le programme, par contre des améliorations doivent être apportées pour assurer une mise en œuvre plus efficace. Les étapes essentielles seront détaillées par l'entremise d'études antérieures et d'études de cas réelles.

\section{Qu'est-ce qu'un PAC?}

La planification des améliorations communautaires, qui fait partie des nombreux outils de planification communautaire durable offerts par la Loi sur l'aménagement du territoire, peut aider les municipalités à relever certains de ces défis, car elle permet de planifier et de financer des activités d'aménagement qui utilisent, réutilisent et réhabilitent efficacement les 
terrains, les bâtiments et l'infrastructure. (Ministère des Affaires municipales et du Logement, 2008)

En outre, le PAC offre des programmes incitatifs aux entreprises situées dans certaines zones désignées afin d'améliorer ou rénover leurs propriétés. « La Loi sur l'aménagement du territoire définit une «zone d'améliorations communautaires » comme une municipalité ou zone située dans une municipalité où, de l'avis du conseil, des améliorations communautaires sont souhaitables pour des raisons de vétusté, de délabrement, de surpeuplement ou d'aménagement défectueux » (Municipalité de Russell, 2015). Ce programme devient de plus en plus populaire chez les municipalités puisqu'il permet de revitaliser les centres-villes, développer les zones sous-utilisées et améliorer la qualité de vie des résidents pour ainsi assurer la durabilité économique de la région. «Toutes les municipalités ontariennes doivent se construire, se renforcer ou se transformer pour [...] répondre aux besoins futurs de leur population à titre de collectivités durables offrant une excellente qualité de vie » (Ministère des Affaires municipales et du Logement, 2008).

Plusieurs programmes incitatifs existent, soit sous forme de subvention, prêt ou aide fiscale. Par exemple, la municipalité de Russell offre présentement huit différents programmes, tels que : amélioration de la façade, amélioration des enseignes, amélioration de l'accessibilité, conversion d'usage résidentiel à usage commercial, stationnement et aménagement paysager, conception architecturale ou d'ingénierie, application municipale / rabais aux frais de permis et la réduction des taxes. Bien qu'il en existe d'autres, chaque municipalité peut adapter les programmes qu'elles offrent selon ses préférences et besoins.

\section{Comment assurer une mise en œuvre réussie?}

Même si la mise en œuvre d'un projet constitue un lourd engagement pour toute organisation, cette étape reste primordiale à son succès. Si le projet reste sur le bureau et aucune action est assignée, il sera impossible pour le projet de porter fruit à la communauté et au gouvernement municipal. Ci-dessous, vous trouverez les éléments essentiels pour assurer une implantation réussie du PAC.

\section{Promotion}

Une fois la rédaction du PAC complétée, il demeure important de promouvoir le programme au sein de la communauté. Pour ce faire, le développement d'une stratégie marketing spécifique au PAC sera nécessaire pour venir différencier le programme et donner une identité propre. Cette stratégie permettra à plus de gens possibles de connaître le programme et d'en bénéficier. 
Afin d'augmenter la visibilité du programme, il est avisé de faire la publicité de différentes façons, soit par l'entremise de médias sociaux, journaux, conférences de presse, brochures, etc.. Sans la promotion du programme, il n'y aura aucune façon pour les gens de la communauté de connaître le programme qui leur en est disponible. De nos jours, il devient de plus en plus difficile de faire passer un message aux gens dû à la surcharge d'horaires personnels et professionnels. Voilà pourquoi il est important d'afficher les publicités sous différents formats tels qu'en ligne, copie papier, etc.

Une autre façon de promouvoir les bienfaits du programme est de rendre les histoires à succès disponibles aux yeux de la communauté. Par exemple, aussitôt que la première subvention est accordée et que le projet de revitalisation est complété, publier des photos avant et après demeure une excellente méthode pour faire connaître le PAC auprès de la population. En plus, le fait d'inclure des citations d'entrepreneurs satisfaits du plan d'améliorations communautaires est une autre façon d'augmenter la promotion du programme. Ceci permet aux participants potentiels d'être confiants dans le $\mathrm{PAC}$ et de les encourager davantage à participer.

Tel que stipulé dans le rapport du personnel de la ville de Waterloo, l'expérience acquise dans plusieurs municipalités a montré qu'une mise en œuvre efficace et la promotion des programmes incitatifs se traduiraient par un PAC plus efficace (Mitchell, 2015).

\section{Observation}

Un autre élément qui demeure essentiel à une implantation réussie du PAC est l'observation continue. Chaque programme que le gouvernement municipal offre à sa communauté d'entreprises doit être observé régulièrement. Tout d'abord, les objectifs donnés pour chaque programme doivent être atteints de façon à ce que les résultats du PAC soient bénéfiques pour l'ensemble de la communauté. Pour la municipalité de Russell, des lignes directrices sont recommandées pour chacun des programmes incitatifs. Ces normes de conception et repères de développement assurent que les participants du programme suivent les recommandations de la municipalité tout en ayant une certaine flexibilité pour assurer de la variété. Cependant, les participants doivent tout de même remplir leurs engagements en assurant que le résultat final est le même tel que proposé dans l'application du PAC. De plus, l'observation des avantages que chaque programme apporte à la communauté est primordiale pour assurer que le PAC atteint son objectif principal, soit de revitaliser les centres-villes. La diversification des programmes du PAC assurera une panoplie d'améliorations en aménagement paysager, enseigne et accessibilité, pour en nommer quelques-uns. « Le conseil municipal se réserve le droit de limiter l'accès à certains programmes dans les cas où il aura déterminé que certaines subventions sont redondantes et ne représentent pas une utilisation adéquate des ressources [...] » (Municipalité de Russell, 2015). 


\section{Exécution}

La façon que le projet est exécuté demeure une étape très importante aux yeux des participants. Lorsqu'une application est soumise au département responsable, l'équipe de gestion doit revoir l'application dans un temps satisfaisant pour assurer l'intégrité du projet. Pour assurer une livraison raisonnable des résultats à l'appliquant, il demeure primordial de former les gens de l'équipe de gestion du PAC. Ceci va engendrer une révision juste et rapide de l'application. Si l'appliquant ne reçoit pas une confirmation que sa demande a été acceptée dans un délai raisonnable, il ne sera pas tenté de promouvoir le programme auprès de ses proches. «Des clients satisfaits contribueront à votre entreprise pendant des années grâce à leurs achats et aux recommandations et références qu'ils feront au sujet de votre entreprise » (Info Entrepreneurs, 2009). Il reste donc important d'offrir un bon service par rapport au programme pour assurer sa réussite et sa continuité.

\section{Révision}

Selon les besoins, un suivi au niveau de la performance du PAC et des ajustements au programme permettront également d'assurer un programme efficace. Pour assurer un suivi de la performance, une évaluation régulière des indicateurs de performance comme le montant total de subventions accordées chaque année, l'évaluation des propriétés, la satisfaction des demandeurs, etc. est nécessaire. Suite à l'observation et l'exécution du programme, faire des ajustements est une étape que plusieurs municipalités ne doivent pas oublier. Que ce soit au niveau des programmes incitatifs offerts, des politiques, de l'administration ou du financement, les ajustements permettront au gouvernement municipal d'atteindre ses objectifs en lien avec le PAC (Ministère des Affaires municipales et du Logement, 2008). Pour la municipalité de Russell « [1] e conseil municipal peut faire tout ajout, révision ou changement au présent PAC, pendant les cinq premières années du programme ou par la suite, en fonction de ses objectifs et des résultats obtenus » (Municipalité de Russell, 2015). Dans presque tous les domaines, apporter des modifications améliore souvent le produit ou service. C'est le même cas pour le plan d'améliorations communautaires.

Étant donné que la promotion du PAC est l'élément essentiel qui vient en premier lieu pour assurer sa réussite au niveau de l'implantation, deux études de cas seront présentées pour venir identifier les facteurs critiques au succès de sa mise en œuvre.

\section{Étude de cas 1 - Spruce the Bruce}

Spruce the Bruce appuie les efforts de la communauté locale pour faciliter les plans de revitalisation du centre-ville à long terme, regroupant les parties prenantes pour développer la capacité de la communauté et aider à la 
politique stratégique et à l'investissement en capital. Le programme offre aux communautés les ressources nécessaires pour élaborer des stratégies et des méthodes de mise en œuvre pour maintenir et développer des zones commerciales saines [...] (Spruce the Bruce, 2017).

Ce projet, qui est géré par les Comtés de Bruce depuis maintenant huit ans, sert à construire de meilleurs centres-villes pour ses résidents et visiteurs. Situé au sud-ouest de l'Ontario, Spruce the Bruce semble comprendre les éléments essentiels pour assurer une implantation réussie de son PAC. En plus d'offrir un programme de développement communautaire aussi appelé un plan d'améliorations communautaires (Spruce the Bruce), les Comtés de Bruce ont tout récemment en mars 2017 élargi leurs subventions disponibles pour inclure de l'aide en développement d'entreprises (Business Development) ainsi qu'en promotion et marketing (Explore the Bruce). En élargissant leur programme, non seulement les commerçants ont accès à des subventions, mais également à des outils de supports pour faire grandir leur entreprise. Cette diversification de services disponibles engendrera une meilleure promotion du programme puisque les propriétaires d'entreprise auront plus de matériels à faire partager à la communauté.

Une stratégie marketing spécifique à Spruce the Bruce a été développée de façon impeccable pour assurer une excellente visibilité du programme. Un site Internet a spécifiquement été créé pour annoncer le programme à la communauté et pour démonter ses différentes facettes. En ayant un site Internet facile à naviguer, cela augmente la circulation sur les différentes pages, ce qui aide grandement à promouvoir le programme. Pour venir informer les différentes générations, Spruce the Bruce est également présent sur les médias sociaux, tels que Facebook et Twitter. En plus de leur présence accrue, Spruce the Bruce rend ses histoires à succès disponibles sur son site Internet et médias sociaux en affichant des photos avant et après des projets complétés. Ceci démontre aux gens de la communauté et à ceux de l'extérieur les bienfaits du programme. Cette méthode apporte également un sentiment de fierté pour les participants du programme.

En ayant comme but principal de créer des destinations dans ces centres-villes, le programme Spruce the Bruce offre une gamme de services complets pour les entrepreneurs de la région. Que ce soit un soutien dans la recherche de politiques, dans le développement d'un plan d'action, dans les services de conception ou dans l'obtention de subventions, le programme comprend tous les aspects nécessaires pour assurer sa réussite au niveau de la promotion. Des trousses d'outils ont même été développées pour chacune des différentes communautés de Bruce afin de donner une identité propre à chacune des régions. Ces trousses, qui incluent un plan de revitalisation se concentrant sur la communauté en question, comprend l'information nécessaire pour informer un appliquant. Les prochaines étapes sont clairement identifiées dans chacune des trousses pour aider aux propriétaires d'entreprises d'aller de l'avant avec la demande de services ou de subventions sans aucun souci. Plus que l'information est véhiculée de façon claire et précise, 
plus les gens ont tendance à continuer avec le projet et c'est exactement ce que Spruce the Bruce a réussi à comprendre. Aussi, il y a plusieurs pages infographiques dans les trousses qui expliquent davantage chacune des facettes des subventions (voir figure 1). «Des études ont révélé que 90\% des informations transmises au cerveau sont visuelles » (Inspired Mag, 2015), voilà pourquoi l'utilisation de représentations créatives et illustratives devient une méthode de marketing efficace. Lorsque la publicité des différents programmes est attirante à l'œil, la promotion du programme est plus efficace. Bref, Spruce the Bruce comprend parfaitement comment réussir au niveau de la promotion de son plan d'améliorations communautaires.

\section{Figure 1. Spruce the Bruce - Downtown Facade Improvement Guidelines}

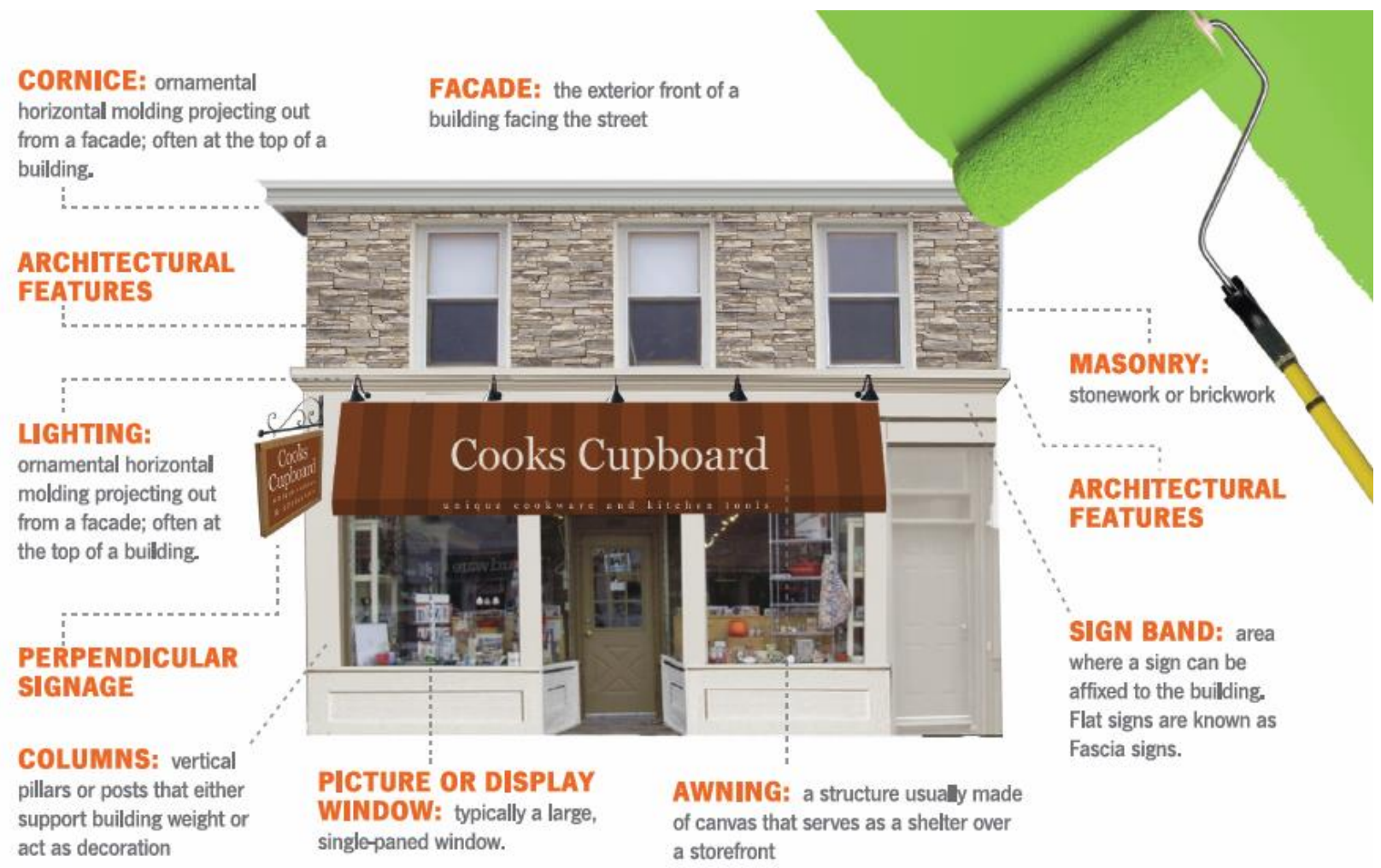

Source : Bruce County. (2017). Downtown Facade Improvement Guidelines. Retrieved from http://www.brucecounty.on.ca/assets/departments/planning/files/STB\%20Documents/FacadeGrants-Flyer-HR.pdf

\section{Étude de cas 2 : Brantford}

La ville de Brantford a développé un plan d'améliorations communautaires offrant des incitations pour supporter leur communauté d'entreprises vibrantes. Situé au cœur du sud de l'Ontario, Brantford comprend l'importance de développer des politiques de marketing pour promouvoir l'adoption de ses programmes incitatifs. Tout d'abord, un document comprenant l'information essentielle du PAC est disponible sur leur site Internet. En plus d'inclure les informations de chaque programme disponible et les raisons principales pour ouvrir une entreprise à cet endroit, Brantford a également inclus des citations d'entrepreneurs de la région 
qui ont été satisfaits du PAC. Par exemple, Jenna Garbedian, de The Works Gourmet Burger Bistro indique que : «En tant que nouveau restaurant sur un délai serré pour ouvrir, la subvention du centre-ville a été la partie la plus simple de tout le processus d'ouverture de notre entreprise » (City of Brantford, 2017). En ayant des citations positives d'entrepreneurs locaux, le programme devient plus réel aux yeux de la communauté et permet d'afficher les avantages que les gens ressortent du programme. De plus, les propriétaires potentiels de recevoir la même subvention deviennent plus confiants lorsqu'ils reçoivent confirmation qu'une autre entreprise a réussie.

Selon le manuel de planification des améliorations communautaires 2008 du Ministère des Affaires municipales et du Logement, une des méthodes pour promouvoir la participation aux programmes est par l'entreprise de «partenariats avec les groupes communautaires, les associations d'entreprises locales et les autres paliers d'administration » (Ministère des Affaires municipales et du Logement, 2008). La ville de Brantford a développé des partenariats avec le Casino OLG, l'Université Wilfrid-Laurier et d'autres établissements postsecondaires pour aider à promouvoir son programme $\mathrm{PAC}$ autant que possible. Ces partenariats permettent à la ville de Brantford de créer des liens et d'assurer une croissance soutenue qui vient considérablement améliorer la réussite du plan d'améliorations communautaires. « La ville s'est également associée à Downtown Brantford BIA pour aider à créer une communauté commerciale dynamique pour les étudiants, les citoyens de Brantford et les communautés environnantes » (City of Brantford, 2017). Avoir accès à de différents réseaux et de nouvelles compétences génère de nouvelles idées et pousse à l'innovation. La ville de Brantford peut donc obtenir l'expertise de ses partenaires pour continuellement améliorer son Plan d'améliorations communautaires. «Selon le magazine Harvard Business Review, [...] une entreprise qui s'engage dans des processus de relations collaboratives peut voir ses bénéfices progresser de 25\% à 95\% » (Côté, 2017). Les méthodes promotionnelles que la Ville de Brantford utilisent semblent grandement rendre leur Plan d'améliorations communautaires plus efficace.

\section{Municipalité de Russell - Recommandations}

La municipalité de Russell, situé à 25 minutes au sud-est d'Ottawa, offre le programme du Plan d'améliorations communautaires dans certaines zones des villages d'Embrun et Russell depuis janvier 2016. La rédaction du programme a été faite avec précision et selon les besoins des centres-villes. Cependant, il est nécessaire d'apporter des améliorations au programme pour assurer une mise en œuvre améliorée.

Durant la première année du PAC, la municipalité de Russell a seulement reçu trois applications d'entreprises situées dans les zones du programme. En début 2017, l'équipe a donc concentré ses efforts à développer une fiche publicitaire comprenant l'information générale du programme (voir figure 2). Elle a été distribuée par la poste à chacun des propriétaires d'édifices inclus dans 
les zones du Plan d'améliorations communautaires pour assurer que chacun soit informé du programme. De plus, un dîner-conférence a été offert à la population pour discuter des détails du programme. Des photos avant et après du bâtiment qui a reçu le premier chèque en lien avec la subvention pour l'amélioration de la façade ont été ajoutées à notre page Facebook pour promouvoir le programme à la communauté. Ces méthodes promotionnelles ont certainement aidé à faire connaitre le PAC aux résidents de la municipalité de Russell puisque de janvier à mai 2017 nous avons déjà reçu cinq applications. De plus, l'équipe reçoit beaucoup plus de demandes d'information que l'an dernier, ce qui nous indique que les gens ont pris connaissance de l'existence du programme. Au niveau de la promotion, la municipalité de Russell a suivi la plupart des éléments essentiels pour assurer une mise en œuvre réussie. Le développement d'une stratégie marketing améliorerait davantage la promotion du programme puisque les gens pourraient associer plus facilement chaque publicité au PAC. Aussi, développer des trousses d'outils similaires à Spruce the Bruce en incluant des citations comme a fait la ville de Brantford viendrait informer d'autant plus les participants potentiels. Incorporer ces outils à notre site Internet en ligne attirerait également de nouvelles entreprises de l'extérieur.

\section{Figure 2. Municipalité de Russell Plan d'améliorations communautaires}

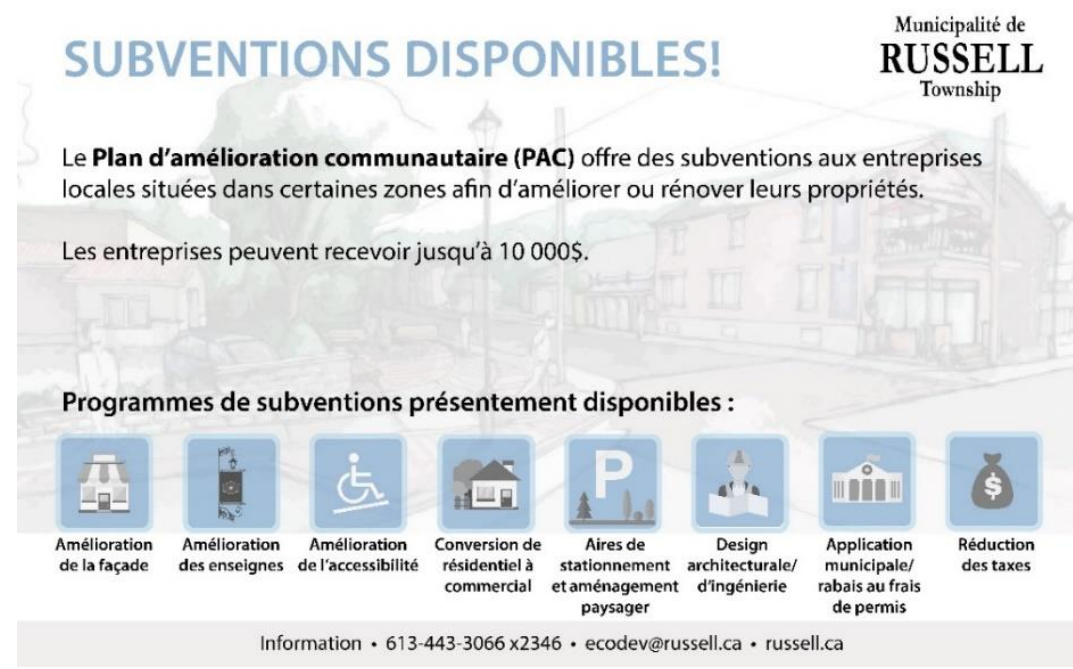

Source : Municipalité de Russell, Plan d'améliorations communautaires

Au niveau de l'exécution du PAC, plusieurs améliorations doivent être apportées puisque c'est une étape très importante aux yeux des participants. Tout d'abord, les gens de l'équipe de gestion du PAC ont été formés par le consultant qui a développé le programme, cependant la livraison des résultats est plutôt longue. Plus précisément, une fois que notre équipe reçoit l'application complète, chacun des membres doit la revoir selon nos lignes directrices recommandées. Ensuite, le chef de l'équipe revoit les commentaires de chaque membre et rédige une lettre à l'appliquant détaillant le montant qui lui sera attribué selon la demande qui nous a été soumise. Ce processus a déjà pris plus de quelques mois à compléter et ce temps n'est aucunement satisfaisant pour garder l'intégrité du projet. Pour améliorer cette étape, il faudrait fixer des dates limites et 
organiser des réunions afin que les membres de l'équipe puissent réduire le temps d'attente pour l'appliquant. Ceci assurera un client satisfait qui par la suite augmentera nos chances que celui-ci recommande le programme à ses proches. En apportant ces améliorations à notre Plan d'améliorations communautaires, il sera certain que l'implantation de notre mise en œuvre sera plus efficace, ce qui engendrera de meilleurs résultats et une revitalisation réussie de nos centresvilles.

\section{Conclusion}

Les centres-villes, étant le cœur des communautés, doivent satisfaire les résidents en plus d'attirer des visiteurs afin que les communautés puissent grandir économiquement. Pour assurer une implantation réussie du PAC, les éléments essentiels étant la promotion, l'observation, l'exécution et la révision doivent être exécutés minutieusement. Dans ce rapport, nous avons appris que les méthodes promotionnelles demeurent importantes à une implantation réussie du Plan d'améliorations communautaires. Suite aux deux études de cas mentionnées ci-haut, nous pouvons confirmer que la promotion est la première étape qui fait en sorte que le programme réussit d'une part pour sa communauté. Une fois cette étape survenue, il demeure indispensable de se concentrer sur les prochains éléments fondamentaux pour assurer une implémentation réussie du Plan d'améliorations communautaires.

Selon le Plan stratégique de développement économique 2014-2016 de la municipalité de Russell, l'un des objectifs pour soutenir la croissance de la communauté est de «promouvoir l'attrait de la population par le biais d'un développement urbain bien planifié, en phase avec les stratégies de Prescott-Russell pour fonder un lieu d'une qualité exceptionnelle » (Municipalité de Russell, 2015). Une mise en œuvre du Plan d'améliorations communautaires fondée sur les éléments essentiels à la réussite viendra certainement améliorer une communauté pour le meilleur.

\section{Biographie de l'auteur}

Vanessa Carrière est l'adjointe administrative au département de développement économique de la municipalité de Russell depuis mai 2015. Elle détient depuis 2014 un baccalauréat en sciences commerciales, option en Management de l'École de gestion Telfer de l'Université d'Ottawa et complète présentement sa certification dans le Programme de développement économique de l'Université de Waterloo. En plus de son expérience acquise au niveau municipal, Vanessa a également travaillé au niveau gouvernemental dans le Département de la Justice et le Département de Pêches et Océans. 


\section{Références}

Mitchell, J. (2015). Staff Report Growth Management - Proposed Uptown Community Improvement Plan. Retrieved from http://www.waterloo.ca/en/contentresources/resources/government/Uptown_CIP_propose d_plan.pdf

Côté, E. (2017, Mai). Définir l’innovation. Le Droit Affaires, volume 8(5), 40 pages.

City of Brantford. (2017). Downtown Brantford Revitalized. Retrieved from http://www.brantford.ca/Projects\%20\%20Initiatives\%20\%20Downtown/DowntownIncen tivesBrochure-WEB.pdf

Info Entrepreneurs. (2009). Gérer votre service à la clientèle. Retrieved from http://www.infoentrepreneurs.org/fr/guides/bl---gerer-votre-service-a-la-clientele/

Inspired Mag. (2015). Why infographics are so important to your content marketing strategy. Retrieved from http://inspiredm.com/why-infographics-are-so-important-to-your-contentmarketing-strategy/

Ministère des Affaires municipales et du Logement. (2008). Manuel de planification des améliorations communautaires 2008. Retrieved from http://www.mah.gov.on.ca/Page3487.aspx

Municipalité de Russell. (2015). Community Improvement Plan. Retrieved from http://fr.russell.hosted.civiclive.com/UserFiles/Servers/Server_3708999/File/Hôtel\%20de \%20ville/Projects\%20en\%20cours/Plan\%20d'amélioration\%20communautaire\%20et\%20 patrimoines/26871_Russell_CIP_Doc_Print_04\%20annexe\%20A\%20by-law.pdf

Spruce the Bruce. (2017). Downtown Facade Improvement Guidelines. Retrieved from http://www.brucecounty.on.ca/assets/departments/planning/files/STB\%20Documents/Fac adeGrants-Flyer-HR.pdf

Spruce the Bruce. (2017). Spruce the Bruce Community Development Program. Retrieved from http://www.brucecounty.on.ca/business/spruce-the-bruce.php 